\title{
The Synaptic Drive from the Spinal Locomotor Network to Motoneurons in the Newborn Rat
}

\author{
Jean-René Cazalets, ${ }^{1}$ Michel Borde, ${ }^{2}$ and François Clarac ${ }^{1}$ \\ ${ }^{1}$ CNRS, NBM, 13402 Marseille Cedex 20, France, and Laboratorio de Neurofisiologia, Facultad de Medicina, Flores \\ 2125, 11800 Montevideo, Uruguay
}

The nature of the synaptic drive from the locomotor spinal network onto the motoneurons was studied in the newborn rat. For this purpose, an in vitro isolated spinal cord preparation of newborn rat was used. The recording chamber was partitioned with Vaseline walls to separate the L1/L2 lumbar segments, in which the spinal locomotor network is located, from the motoneurons in the lower lumbar segments. Locomotor-like activity was induced by bath-applying a mixture of serotonin and NMDA to segments L1/L2. In this way, the synaptic activity could be modified at the lower lumbar level without affecting the motor pattern. The drive elicited onto the motoneurons during sequences of locomotor-like activity, which was monitored by performing intracellular recordings, consisting of an inhibitory component followed by an excitatory component. The inhibitory synaptic volley was reversed at a membrane potential of $-60 \mathrm{mV}$ with $\mathrm{K}$ acetate electrodes, whereas it was shifted toward positive values with $\mathrm{KCl}$ electrodes. The glycinergic blocker strychnine, bath-applied to segments L3/L5, blocked the inhibitory drive without affecting the rhythmic activity, whereas it disrupted the locomotor-like activity when bathapplied to segments L1/L2. The inhibitory part of the drive was more sensitive than the excitatory part to changes in the membrane potential. The excitatory phase was mixed and consisted of an NMDA and a non-NMDA component, which were sensitive to 2-amino-5-phosphonovaleric acid and 6-cyano-7-nitroquinoxaline-2,3-dione, respectively. It was concluded that the locomotor network located in segments $L 1 / L 2$ sends a biphasic projection to the various groups of motoneurons located along the lumbar spinal cord.

Key words: electrophysiology; spinal cord; locomotion; motoneuron drive; glycine; excitatory amino acids
During locomotor activity in mammals, the motoneurons are rhythmically depolarized and produce phasic bursts of action potentials which, in turn, elicit muscle contractions and limb movements. Schematically, two main components located at the lumbar spinal level can be described to take part in these motor rhythm generation processes: (1) the premotoneuronal locomotor spinal network, which is also called the central pattern generator (CPG); and (2) the motoneurons onto which all of the integrated information converges (Grillner, 1981). Although a considerable amount of data has been collected on the synaptic mechanisms responsible for this rhythmic behavior (for review, see Jordan, 1983, 1991; Shefshick and Jordan, 1985; Perret, 1986; Roberts et al., 1986; Grillner and Matsushima, 1991), one question still remains regarding the relative contributions of these various elements, i.e., those of the premotoneuronal network on the one hand and the output motoneuronal compartment on the other hand. This gap in our knowledge has been attributable mainly to technical limitations that made it impossible to study these two elements separately. Using an in vitro isolated brainstem/spinal cord preparation of newborn rat, we recently demonstrated (Cazalets et al., 1995) that it is possible, by building a Vaseline wall at the lumbar segmental level (L2/L3), to separate physically the CPG itself, which is located in the rostral part of the lumbar cord, from its target motoneurons located in the caudal part.

Received Feb. 28, 1995; revised Sept. 15, 1995; accepted Sept. 22, 1995.

This research was supported by a grant from NATO and by INSERM Grant 920803. Many thanks to R. Navarro for nursing the pups. The authors thank Dr. Jessica Blane for revising the English manuscript.

Correspondence should be addressed to Dr. Jean-René Cazalets, CNRS, NBM, 31 Chemin Joseph Aiguier, BP 71, 13402 Marseille Cedex 20, France.

Copyright $(C) 1995$ Society for Neuroscience $0270-6474 / 95 / 160298-09 \$ 05.00 / 0$
Under these conditions, locomotor-like activity can be initiated by directly bath-applying activatory transmitters to the rostral area only. The CPG, in turn, will rhythmically activate the motoneurons located in the lower part of the spinal cord. We have established moreover that some connections, between the rhythmic network located at the L1/L2 level and the motoneurons, are monosynaptic. Consequently, it is now possible with this method to study separately and to identify the various components that constitute the synaptic drive exerted by the CPG on the motoneurons. In this study, we first demonstrate that the motoneuronal drive consists of two components, one inhibitory and the other excitatory. In the second step, we identify the transmitters involved in this biphasic activation. The inhibitory component, which is blocked by strychnine, appears to involve glycine, whereas the excitatory component may be mediated by excitatory amino acids via the activation of both NMD $\Lambda$ and non-NMDA receptors. The respective contributions of the two components to the genesis of rhythmic discharge in motoneurons are discussed, and the results are compared with those obtained using other vertebrate locomotor models.

\section{MATERIALS AND METHODS}

Experiments $(n=28)$ were performed on newborn Wistar rats aged 1-4 d. The rats were anesthetized with ether before decapitation. The dissection procedure adopted and the experimental methods used to prepare the isolated in vitro brainstem/spinal cord have been described extensively in previous papers (Sqalli-Houssaini et al., 1991, 1993a; Cazalets et al., 1992).

Partitioning of the spinal cord and intracellular recordings. Walls were built between the various parts of the bath with a syringe containing solid Vaseline, which was deposited with a fine needle $(0.5 \mathrm{~mm})$. The criteria used to ensure the water tightness of the wall were the same as those 
A

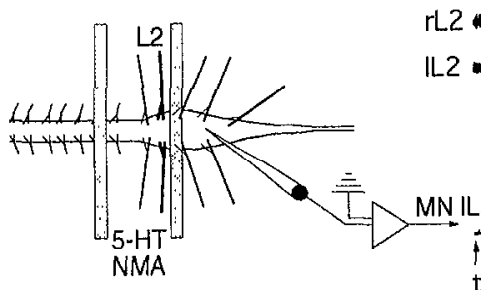

B2

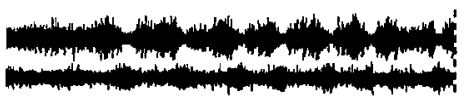

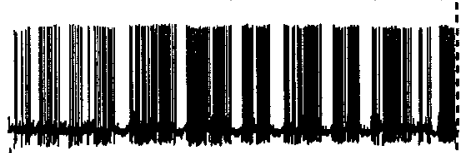

t42

B1
5

B3

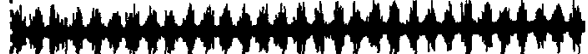

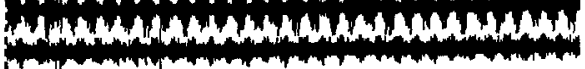

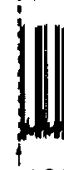

t180

C
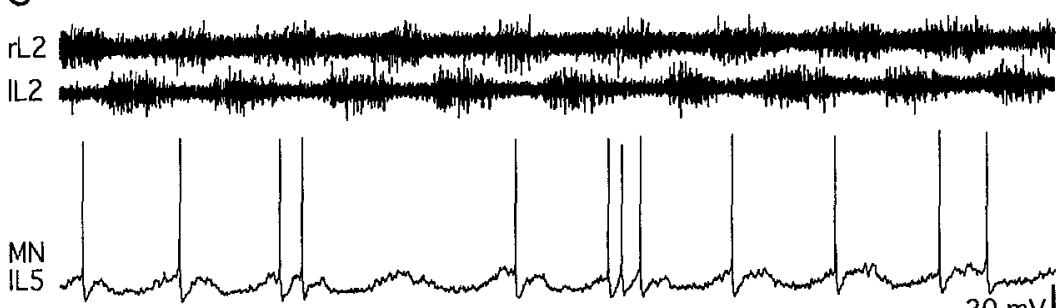

Figure 1. Induction of locomotor-like activity in a partitioned spinal cord. $A$, Diagram of the experimental preparation. A Vaseline wall was built at the T13 and L2 level (gray bars) to superfuse the rostral lumbar cord separately from the caudal lumbar cord. A mixture of 5-HT and NMDA (NMA) was bath-applied to segments L1/L2. Locomotor-like activity was monitored by performing extracellular recordings on the various lumbar ventral roots (L1-L5). In this figure, only the activity in L2 is displayed. An intracellular recording was performed on one motoneuron in segment L5. $B$, When the 5-HT/NMD $\Lambda$ mixture reached the $\mathrm{L} 1 / \mathrm{L} 2$ segments $\left(t_{0}\right.$ : membrane potential, -62 $\mathrm{mV}$ ) the tonic activity increased in the ventral roots, whereas the motoneuron was gradually depolarized. $B 1$ and $B 2$ are continuous recordings. Within $3 \mathrm{~min}\left(t_{180}: \mathrm{mem}-\right.$ brane potential, $-46 \mathrm{mV}$ ), the system became rhythmically active and the intracellularly tested motoneuron emitted bursts of action potentials in phase with the activity in rL2. $C$, Expanded time recording. The membrane potential of the motoneuron oscillated during locomotor-like activity, and action potentials were produced at the crest of the oscillation (membrane potential at the bottom, $-58 \mathrm{mV}$ ). $B$ and $C$ are based on two different neurons. $r L$ and $l L$, right and left lumbar ventral root; $M N$, motoneuron. described previously (Cazalets et al., 1995). Vaseline barriers were built at levels T12/T13 and L2/L3 (unless otherwise specified in the text) to superfuse the low lumbar spinal cord (L3, L4, and L5 segments) separately from the upper spinal segments (T13, I.1, and L2 segments), as shown in Figure $1 A$. This made it possible to superfuse selectively the two compartments and to influence their activity by applying salines and/or drugs with various ionic compositions. For details of the drug superfusion procedure, see Sqalli-Houssaini et al. (1991) and Cazalets et al. (1992, 1994). In all of the experiments described here, locomotor-like activity was induced by bath-applying a mixture of 5-HT $\left(10^{-4} \mathrm{M}\right)$ and NMDA $\left(2 \times 10^{-5} \mathrm{M}\right)$. The characteristics of the rhythm obtained under these conditions have been described in a previous paper (Sqalli-Houssaini et al., 1993a). Serotonin, 2-amino-5-phosphonovaleric acid (AP-5), NMDA, and strychnine were obtained from Sigma (St. Louis, MO), and 6-cyano7-nitroquinoxaline-2,3-dione (CNQX) and bicuculline were obtained from Research Biochemicals (Natick, MA).

Preparatory to the intracellular recordings, the pia was removed to gain access to the gray matter with glass microelectrodes containing either $2 \mathrm{M}$ $\mathrm{K}$ acetate $(r=40-60 \mathrm{M} \Omega)$ or $3 \mathrm{M} \mathrm{KCl}(r=25-40 \mathrm{M} \Omega)$. The motoneurons were identified by the fact that they produced antidromic potentials in response to ventral root stimulation. Only motoneurons with a resting membrane potential of less than $-60 \mathrm{mV}$ and a spike amplitude of at least $70 \mathrm{mV}$ were used for the tests. Under control conditions, i.e., in a quiescent system in which no drugs were bath-applied to the preparation, the mean membrane potential was $-67 \mathrm{mV} \pm 5 \mathrm{SD}, n=30$ neurons. The motoneurons were identified as flexors or extensors on the basis of the phase of their action potential bursts within the locomotor cycle. We previously observed that during 5-HT/NMDA-induced locomotor-like activity, the burst of action potentials extracellularly recorded in the L5 ventral roots is phase-opposed to the burst of activity recorded in the ipsilateral L2 and L3 ventral roots during one locomotor cycle (Cazalets et al., 1992). This suggested that the flexor and extensor phases could be monitored, and Kiehn and Kjaerulff (1995, in press), by simultaneously recording various muscles and ventral roots in a semi-isolated preparation, recently identified the burst in $L 5$ as constituting the extensor phase. By correlating the activity of the intracellularly tested motoneurons with the activity in these two sets of ventral roots, they could be identified as flexors or extensors.

The EPSPs evoked in the motoneurons in response to dorsal root stimulation were used to monitor the efficiency of the excitatory amino acid antagonists used in this study. It has been established in previous studies that the early monosynaptic EPSP is sensitive mainly to CNQX, whereas the late polysynaptic component is $\Lambda \mathrm{P}-5$-sensitive (Jahr and Yoshioka, 1986; Pinco and Lev-Tov, 1993). In the present study, we also observed that CNQX blocked the early EPSPs, whereas AP-5 blocked the late component. We also noted that in the presence of strychnine, the dorsal root stimulation gave rise to a high-frequency motoneuron discharge (at $\sim 5 \mathrm{~Hz}$, data not shown) that was not related to the locomotor activities.

The error bar displayed in the graphs gives the SD, unless otherwise specified. When the bar does not appear, this means that the SD was smaller than the symbol.

\section{RESULTS}

\section{Biphasic synaptic effects on motoneurons}

Figure 1 gives an example of the changes that occurred when the system was switched from silent to active. The activity of a motoneuron was recorded intracellularly in lower lumbar segments (L3-L5), and a 5-HT/NMDA mixture was bath-applied to segments L1/L2 (see Fig. $1 A$; diagram of the preparation). When the 5-HT/NMDA mixture reached the $\mathrm{L} 1 / \mathrm{L} 2$ level, it induced first tonic and then rhythmic activity in the ventral roots. At the same time, the motoneuron recorded from within L5 underwent a gradual change in its membrane potential, which progressively depolarized before showing a rhythmic spiking pattern, correlated with the onset of activity in the extracellularly recorded ventral roots. The depolarization was sustained by increasing synaptic activity. After this initial phase of tonic activity (Fig. 1B1), which was somewhat irregular, rhythmic 


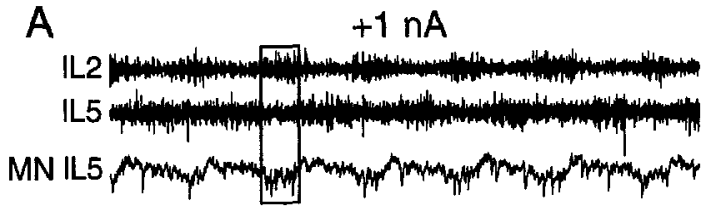

B

Figure 2. Biphasic components of the synaptic drive. $A-C$, Variations in the size and the sign of the inhibitory synaptic volley elicited by injecting current into a motoneuron (membrane potential in $A,-52 \mathrm{mV}$; in $B,-66 \mathrm{mV}$; in $C,-81 \mathrm{mV}) . D$, Change in the am. plitude of the synaptic drive depending on either the current $(D I)$ or the voltage $(D 2)$. At negative values, the total variations (from top to bottom of the oscillation) were measured, because the drive consisted only of depolarizing events. $D 1$ and $D 2$ are recordings obtained on different neurons.

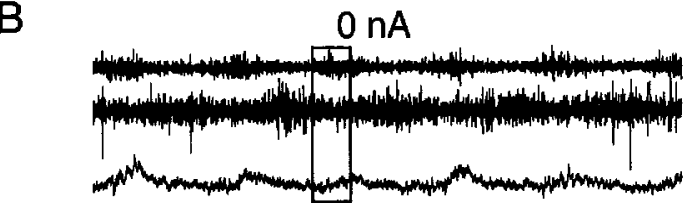

C

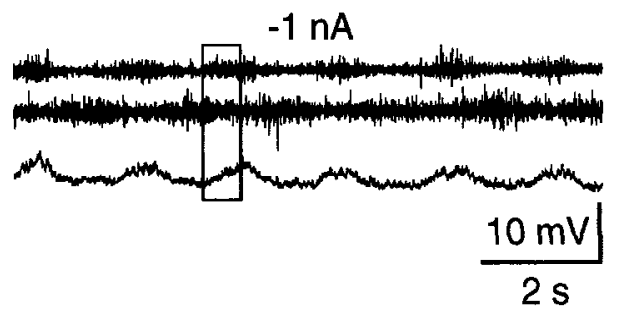

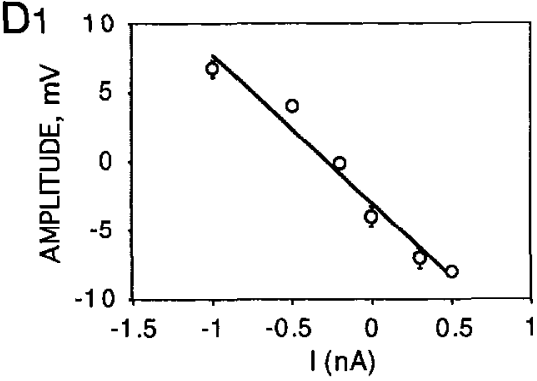

D2

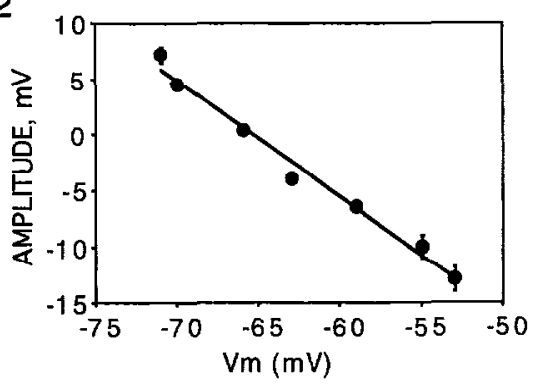

bursts of action potentials occurred in the ventral roots (Fig. 1B2) before the locomotor-like activity was properly established (Fig. $1 B 3)$. Although the recordings shown in Figure $1 B$ are typical from the point of view of the changes with time, the amplitude of the synaptic events was variable and could not be correlated with the type of neurons recorded from (identified as flexors or extensors depending on their firing phase; see Materials and Methods). The initial depolarization ranged between 2 and $20 \mathrm{mV}$ from one neuron to another (mean amplitude $8.3 \pm 6.7 \mathrm{mV}, \mathrm{SD}, n=20$ neurons). The amplitude of the initial depolarization was characteristic of the motoneuron analyzed and was not correlated with any changes in the experimental conditions, because in the same experiment some of the neurons tested at the same level could exhibit large amplitude depolarizations, whereas others did not. Figure $1 C$ shows an expanded trace from one sequence of locomotor-like activity that highlights the changes displayed by the motoneuron membrane potential. The depolarizing phase was followed by a hyperpolarizing phase. The intracellularly recorded motoneuron was emitting action potentials in phase with right lumbar ventral root number 2 (different experiment from that in Fig. $1 B$ ) and, thus, could be classified as an extensor motoneuron (see Materials and Methods for the identification of neurons).

Figure 2 shows that there exists a biphasic component in the synaptic drive during rhythmic discharge. When no current was injected (Fig. 2B), the motoneuron received rhythmic depolarizing inputs that were correlated with the later phase of the burst in the left lumbar ventral root 2 (lL2). When the motoneuron was hyperpolarized by injecting $-1 \mathrm{nA}$ (Fig. $2 C$ ), the size of the drive increased, and when it was depolarized by injecting positive current (Fig. 2A), hyperpolarizing potentials that were correlated with the burst of activity in $1 \mathrm{~L} 2$ appeared; these were followed immediately by a depolarization peak. The voltage dependence of the drive is shown in Figure $2 D$. The change in the drive amplitude was analyzed as a function of either the injected current (Fig. $2 D 1$ ) or the membrane potential (Fig. 2D2), depending on the accuracy with which the bridge could be balanced to determine the membrane potential. The latter parameter was linear in the range of potentials examined $(-80$ to $-40 \mathrm{mV})$, whereas it generally exhibited a rectification at more negative values. As we shall see below, however (Fig. 6D), the various components of the motoneuronal drive did not exhibit the same voltage dependence.

Figure 3 shows that during locomotor-like activity induced in L1/L2, the input resistance of the motoneurons located in the lower lumbar segments decreased, because of synaptic activation arising in $\mathrm{L} 1 / \mathrm{L} 2$. This general decrease in the overall resistance cannot be attributable to the depolarization, because the motoneuron tested in Figure $3 A$ showed only minor changes $(3 \mathrm{mV})$ in the membrane potential (in an oscillating motoneuron, the reference potential was taken at the valley of the oscillation). The mean decrease in the input resistance under these conditions was $-26 \pm 9 \%$ (based on measurements performed on 4 neurons). To test whether part of the changes that occurred during one cycle resulted from passive variations or whether synaptic currents we present during both the inhibitory and excitatory phases, we analyzed the changes in membrane conductance that occurred with time during locomotor-like activity (Fig. 3B). In Figure $3 B 1$, brief hyperpolarizing current pulses were passed through the electrode, and one can see that the resistance changed cyclically with time. Because there were only a few variations in the membrane potential of this neuron, because of the weak amplitude of the synaptic drive, the changes observed in the input resistance could not be attributed to any variations in the membrane potential. We examined these changes more closely by analyzing the variations in the motoneuron input resistance as a function of the phase of the locomotor cycle. For this purpose, we used the protocol described in Figure 3B2. Hyperpolarizing current pulses were applied randomly. The cycle was then normalized on the basis of the activity recorded in right lumbar ventral root number $2(r L 2)$. The period ( $P$, Fig. $3 B 2)$ was calculated from the onset of one burst of spikes to the onset of the following burst. The phase at which the pulse occurred was calculated as its latency in the cycle (l, Fig. $3 B 2$ ) divided by the period. The control resistance was determined when no locomotor activity was elicited. We plotted the changes in resistance during each phase of the cycle (from 0 to 1 ; Fig. 3B3). The cycle consisted of an inhibitory phasc (from 0 to 0.5 ) followed by an excitatory phase (from 0.5 to 1 ). During the depolarizing phase as well as the hyperpolarizing phases, the resistance gradually decreased and then increased. In 
A1 CONTROL

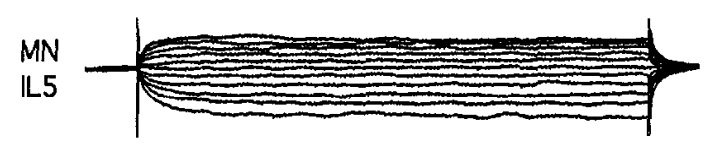

A2 5-HT/NMA on L1/L2

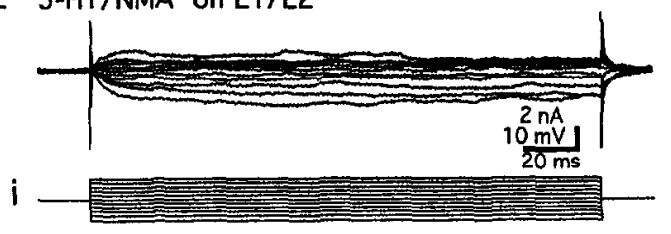

B1
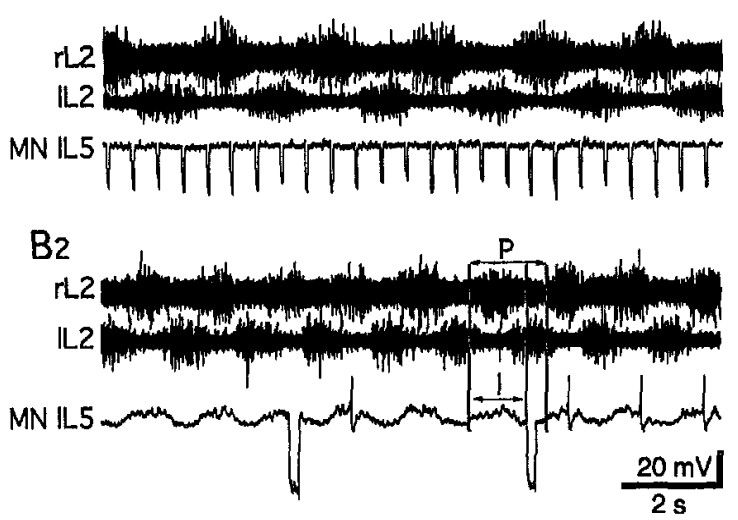

A3

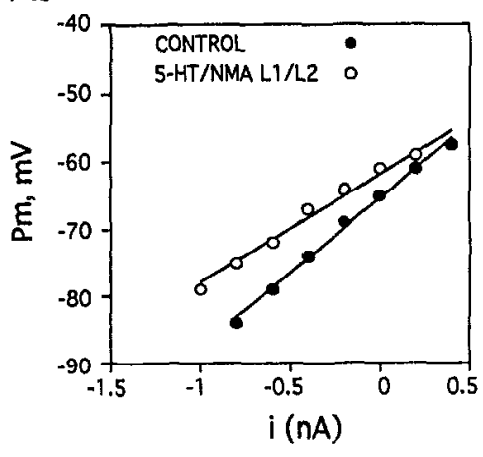

B3

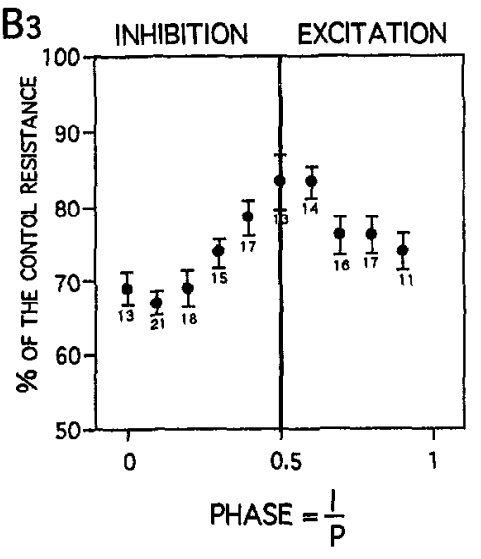

Figure 3. The variations in membrane resistance during locomotor-like activity. Locomotor-like activity was induced by bath-applying 5-HT/NMDA to segments L1/L2. 'The activity of a motoneuron was recorded in segment L5. $A$, Current-voltage relationship of the motoneuron before ( $A 1$; membrane potential, $-65 \mathrm{mV})$ and during $(A 2$; membrane potential, $-61 \mathrm{mV}$ ) a sequence of locomntor-like activity. The I/V curve of the motoneuron $(A 3)$ shows the decrease in membrane resistance elicited by the synaptic effects exerted on the motoneuron during the bath application of 5-HT/NMDA to L1/L2 segments. B1, Variations in the input resistance were monitored by injecting intracellular current pulses $(50 \mathrm{msec}$; $-1 \mathrm{nA} ;$ membrane potential, $-61 \mathrm{mV})$ in the motoneuron (MN $(L 5)$ ). The resulting voltage change varied cyclically with time. $B 2-B 3$, The changes in input resistance were analyzed in relation to the phase of the cycle during which they occurred. Pulses (200 msec, $-1 \mathrm{nA}, 0.2$ $\mathrm{Hz}$ ) were applied repeatedly via the microelectrode. The phase at which the stimulus occurred was calculated as its latency $(l)$ in the cycle divided by the pcriod $(P)$, as indicated in $B 2$. The onset of the activity in $r L 2$ was taken as the baseline. B3, Plot of the resistance changes during one normalized cycle. The control resistance value was determined at rest, i.e., when no drug was bath-applied. The number of measurements is indicated beside each point. The results in $A, B 1, B 2$, and $B 3$ are from different neurons. five out of the six neurons in which this aspect was analyzed, the changes in the resistance were larger during the inhibitory phase than during the excitatory one, whereas in the remaining neuron, no difference was observed between the inhibitory and excitatory phases. The fact that both the hyperpolarizing and the depolarizing phases exhibited cyclic variations suggests, however, that these two phases involved both active depolarization (EPSPs) and hyperpolarization (IPSPs).

\section{The inhibitory component of the synaptic drive onto the motoneurons}

It was observed (Fig. $2 A$ ) that clearly distinguishable IPSPs were elicited in the motoneuron during one cycle of motor activity. We first established the reversal potential of this inhibitory component using potassium acetate electrodes. Under these conditions, the inhibitory phase was found to reverse at $-61.4 \pm 4 \mathrm{mV}, n=$ 10. The inhibition was sensitive to chloride, however. When the motoneurons were impaled with a potassium chloride electrode, there was a shift in the reversal potential toward positive values. Under these conditions, we did not establish the reversal potential directly, because it was too high to be measurable. In terms of the current, the drive was equal to zero when a $-0.2 \pm 0.7 \mathrm{nA}$ current was injected with a potassium acetate electrode, whereas it was equal to zero when a $2.3 \pm 1 \mathrm{nA}$ current (determined by extrapolation) was injected with a potassium chloride electrode ( $n=3$ in each case).

We then studied the nature of the transmitter involved in the inhibitory transmission and observed that it was sensitive to the glycinergic blocker strychnine. Preliminary experiments with strychnine showed, however, that this substance greatly modified the rhythm. For this reason, we first studied its action on the overall motor pattern (Fig. 4). Bath application of strychnine alone to the whole spinal cord (in 6 experiments) at concentrations ranging from 3 to $5 \times 10^{-6} \mathrm{M}$ elicited seizures consisting of large bursts of activity with a very long cycle period (more than 30 sec; Fig. 4Al). These long bursts of activity consisted of highfrequency bursts of action potentials (between 3 and $5 \mathrm{~Hz}$ ), which were in-phase in all of the ventral roots (Fig. 4A2). We then studied the effects of strychnine during locomotor-like activity (Fig. 4B,C). Under control conditions, bath application of 5-HT/ NMDA induced a regularly alternating pattern in the various roots recorded (Fig. $4 B 1, B 2)$. Adding strychnine $\left(>4 \times 10^{-6} \mathrm{M}\right)$ to the saline containing 5-HT/NMDA completely disrupted the motor rhythm, which then consisted only of high-frequency bursts of action potentials (mean $5.6 \pm 1.1 \mathrm{~Hz}, n=5$ experiments). Below $4 \times 10^{-6} \mathrm{M}$, strychnine no longer affected the right and left patterns of alternation.

We then studied the action of strychnine on the synaptic drive when it was perfused only into the motoneuronal pool and not onto the CPG (Fig. 5). In this experiment, a motoneuron was recorded during locomotor-like activity. A ramp of positive current was injected into the motoneuron to make the reversed inhibitory part of the drive visible (Fig. 5A, top of ramp). In the presence of strychnine, the hyperpolarizing component was no longer visible (Fig. $5 B$ ), which suggested that strychnine had blocked the hyperpolarizing events. When strychnine was bathapplied to the lower lumbar segments ( $n=9$ experiments), it always blocked the IPSPs of motoneurons at concentrations ranging from $10^{-6}$ to $5 \times 10^{-6} \mathrm{M}$, whereas we never observed any significant changes in the motor pattern in terms of the period or the phase relationships, even at higher concentrations. When strychnine was bath-applied only to segments L1/L2, however, it disrupted the rhythmic activity completely, in the same way as 


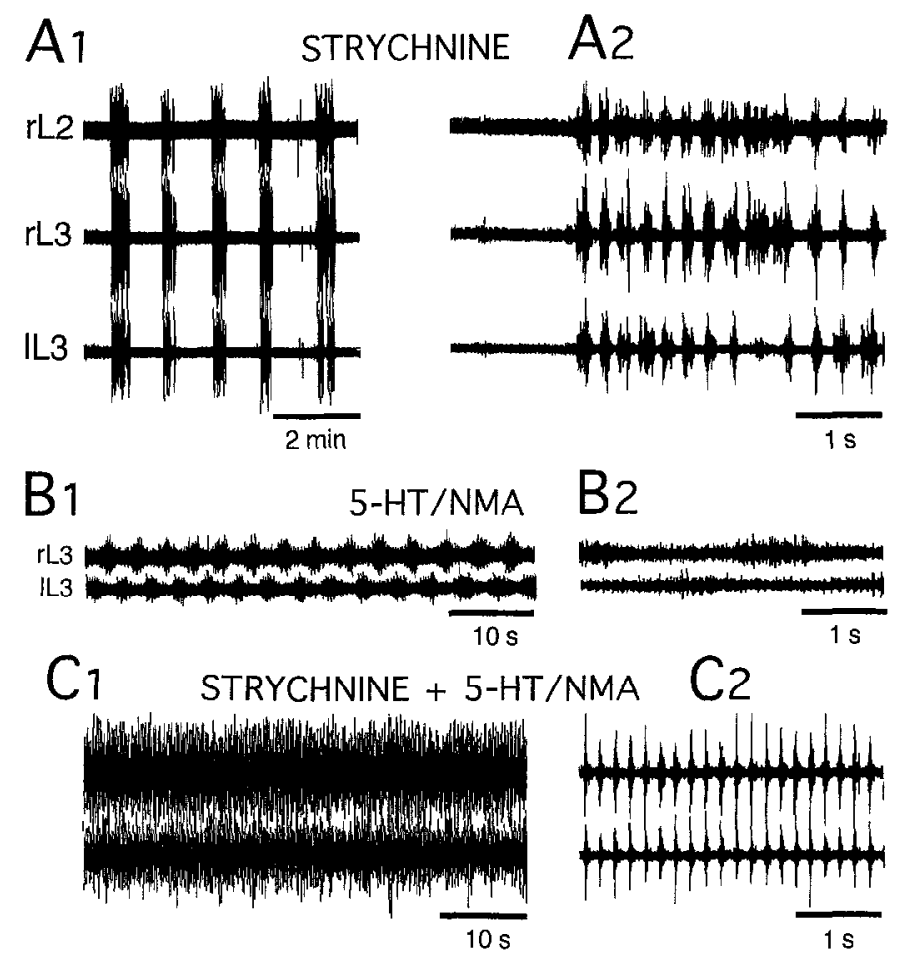

Figure 4. Effects of strychnine on the motor pattern. The left panel shows the motor pattern at a slower time scale, whereas the right panel is an expanded detail of the motor activity. $A$, Bath application of strychnine $\left(5 \times 10^{-6} \mathrm{M}\right)$ alone to the spinal cord elicited large bursts of action potentials with a long interburst interval, which were in-phase in all of the ventral roots $(A I)$. The expanded trace $(A 2)$ shows that each of the slow bursts consisted of high-frequency bursts of action potentials that were in-phase in all of the ventral roots. $B$, Bath application of 5-HT/NMDA elicited an alternating right and left pattern. $C$, Adding strychnine $(5 \times$ $\left.10^{-6} \mathrm{M}\right)$ to the saline containing 5-HT/NMDA completely disrupted the antiphase pattern $(C 1)$ and resulted in a high-frequency discharge (C2) with in-phase bursts in all of the ventral roots. In this experiment, the entire lumbar spinal cord was bathed with the various drugs.

when it was bath-applied to the entire spinal cord (see Fig. 4 legend). In all of the cases tested, the effects of strychnine were difficult to reverse and only partial recovery occurred. On the contrary, when bicuculline, an antagonist of the inhibitory amino acid GABA acting on $\mathrm{GABA}_{\mathrm{A}}$ receptors, was bath-applied to L3/L5 segments, it had no effect on the motoneuron drive.

\section{The excitatory component of the synaptic drive onto the motoneurons}

As we have just seen (Fig. 5), strychnine blocked the inhibitory drive. Because an excitatory component persisted, however, in the presence of strychnine, we performed further experiments under these conditions to study this excitatory drive without interfering with the inhibitory one. In all of these experiments, the efficiency of the excitatory amino acid receptor blockers was tested under our experimental conditions on the EPSPs elicited in the motoneurons by applying primary sensory afferent stimulation (see Materials and Methods).

As shown in Figure 6, the synaptic drive was partly mediated by the activation of NMDA receptors. Figure $6 \mathrm{~A}$ gives an example of a motoneuron recorded from the LS segment in the presence of strychnine, which displayed rhythmic bursting activity during bath application of 5-HT/NMDA to segments L1/L2. Adding AP-5 partly blocked the excitatory drive (Fig. 6B). The effects of AP-5 were investigated in 10 experiments. The average decrease in the drive amplitude was $39.6 \perp 17 \%, n=6$. At the same time, the membrane potential was slightly hyperpolarized $(-3.2 \pm 1.8 \mathrm{mV}$, $n=6$ ) in the presence of AP-5. It was observed that the inhibitory and excitatory parts of the drive did not exhibit the same sensitivity to changes in the current (Fig. 6C). Before strychnine was added to the saline, changes in the injected currents led to great variations in the amplitude (Fig. $6 \mathrm{C}$, open squares), whereas in the presence of strychnine the amplitude varied very little in responses to variations in the current (Fig. $6 C$, filled circles). After adding AP-5, it was observed that the remaining AP-5-resistant part of the drive was still less sensitive to changes in the membrane potential (Fig. 6C, open triangles).

Figure 7 gives evidence supporting the idea that non-NMDA receptors also participate in the excitatory drive. A motoneuron was recorded in the L4 segment in the presence of strychnine to eliminate the inhibitory part of the drive. Under these conditions, adding CNQX to the strychnine saline decreased the excitatory drive (Fig. 7A2). Figure $7 B 1$ gives the various changes in the drive amplitude that occurred in the same neuron during the successive bath application of the various blockers. No change in the drive amplitude occurred after strychnine bath application, because the resting potential was also the equilibrium potential for inhibitory events, as generally happened when recording from a neuron with a potassium acetate electrode (see also Figs, $2 B, 3 B I$ ). CNQX decreased the synaptic drive to a greater extent than AP-5, with a mean decreasc of $64 \pm 21 \%(n=3$ expcriments) and a mean change in the membrane potential of $-4.6 \pm 2.2 \mathrm{mV}(n=4$ experiments). This hyperpolarization, which was also observed with AP-5, suggests that a permanent release of excitatory amino acid occurred at rest. As reported previously (Sqalli et al., 1993), the effects of CNQX were difficult to reverse. In addition, it was observed that when strychnine, AP-5, and CNQX were bathapplied simultaneously to the spinal cord, the synaptic drive completely disappeared. The plot in Figure $7 B 2$ shows that during the bath application of all of these various drugs to the caudal part of the spinal cord, the period value remained unchanged compared with the control value.

\section{DISCUSSION}

The results of this study provide direct evidence that the motoneurons in the isolated spinal cord of newborn rat receive a biphasic locomotor drive, i.e., an inhibitory and an excitatory drive. We have established that the inhibitory part of the drive is probably mediated by glycine, because it is blocked by strychnine, and that the excitatory drive exhibits both an NMDA and a non-NMDA component.

\section{Organization of the spinal locomotor network}

In a previous study, we established that the network responsible for locomotor-like activity in the isolated spinal cord of the newborn rat is located in the upper lumbar segments L1/L2 (Cazalets et al., 1995). In the present study, we used the same methodology with a partitioned spinal cord to study the rhythmic synaptic activation of the motoneurons separately from that of the locomotor spinal network itself. The results confirmed our previous findings, particularly the fact that the main elements responsible for organizing both the period and the phase relationships are not located in the lower lumbar segments. We observed here that strychnine (Fig. 4) disrupted the rhythmic activity when applied either to the entire spinal cord or to segments L1/L2. Furthermore, we previously reported (Cazalets et al., 1992; Sqalli- 


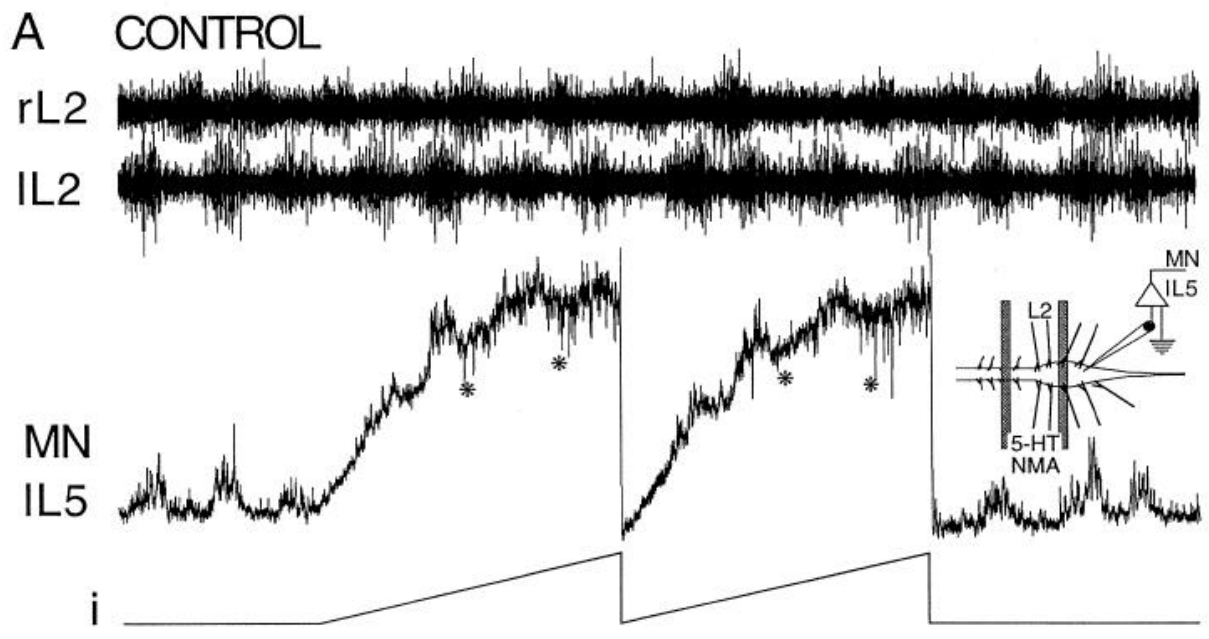

B STRYCHNINE

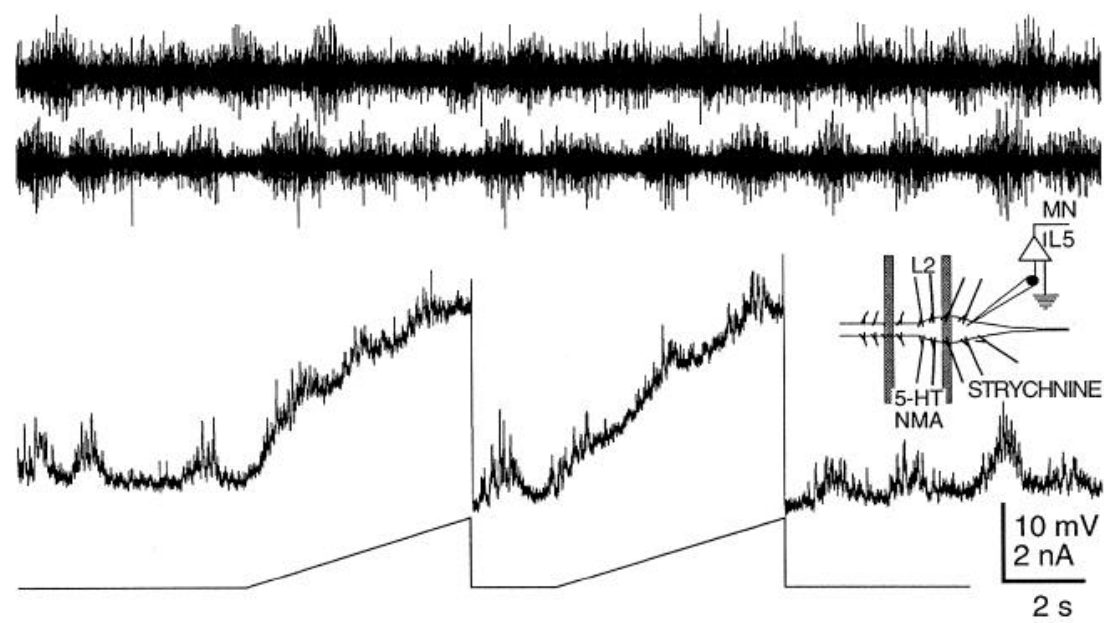

Figure 5. The inhibitory synaptic drive onto motoneurons is blocked by strychnine. Locomotorlike activity was induced by bath-applying 5 -HT/ NMDA to segments L1/L2. The activity of a motoneuron was recorded in segment L5. A, During locomotor-like activity, the motoneuron was rhythmically depolarized (membrane potential at bottom, $-65 \mathrm{mV}$ ). The injection of a ramp of positive current depolarized the neuron and revealed the synaptic hyperpolarizing volley $\left({ }^{*}\right)$. $B$, When strychnine $\left(3 \times 10^{-6} \mathrm{M}\right)$ was bathapplied to the lower segments of the lumbar cord, the hyperpolarizing events no longer appeared.
Houssaini et al., 1993b) that the excitatory amino acids are powerful agents involved in the generation of locomotor-like activity. Correspondingly, the NMDA (AP-5) and non-NMDA (CNQX) receptor antagonists abolished all locomotor-like activity in a dose-dependent manner when bath-applied to the entire lumbar cord (Cazalets et al., 1992; Sqalli-Houssaini et al., 1993b). It was observed, however, in the present study that when all of these various blockers were applied only to the caudal segments of the lumbar cord, they never modified the locomotor-like activity elicited in L1/L2 (Figs. 4-7). This confirms that the main components of the locomotor network are located in the rostral part of the lumbar cord, because if part of the network that organizes the pattern had been located in the caudal area, changes would have been observed in the temporal parameters of the locomotor-like activity occurring under these conditions.

\section{The transmitters involved in the locomotor drive}

Involvement of glycine in the inhibitory drive

We provide in this study the first direct evidence of the nature of the locomotor drive. This was possible because of two previous findings. First, as described previously (Cazalets et al., 1995), we dissociated the CPG from the motoneuron site in our experiments, uncoupling them by superfusing the two compartments separately. Second, by bath-applying to L3/L5 segments a saline containing mephenesin or a high concentration of divalent cations, which suppresses the polysynaptic pathways, the drive onto the motoneuron from the CPG was found to be mainly monosynaptic (Cazalets et al., 1995). These two requirements have to be fulfilled to ensure that the changes observed at a given site, when the drive is blocked by antagonists of the various transmitters, are actually attributable to an action at this site.

The results obtained here using the glycinergic blocker strychnine indicate that glycine plays a crucial role in locomotor processes. First, glycine is involved directly in the premotoneuronal network located in L1/L2, which generates locomotor-like activity. The seizure elicited by strychnine (Fig. $4 A$ ) and the complete disruption of the locomotor-like activity (Fig. $4 \mathrm{C}$ ) occurred only when strychnine was bath-applied to the L1/L2 segments or to the entire spinal cord, never when it was bath-applied only to the motoneurons (i.e., on the caudal part of the lumbar cord; see Figs. 5-7 for examples); this suggests that these effects were attributable primarily to strychnine acting on the locomotor network itself. The possibility that glycinergic interneurons are involved in this inhibitory action was strengthened by the lack of effects observed with GABAergic blockers. In a previous study, we concluded that the inhibitory transmitter GABA could not play a central role within the locomotor network, because its antagonists 
$\mathrm{A}_{1}$

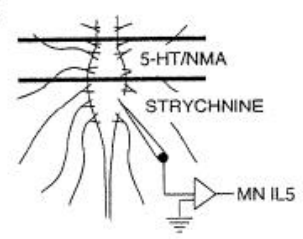

$\mathrm{B} 1$

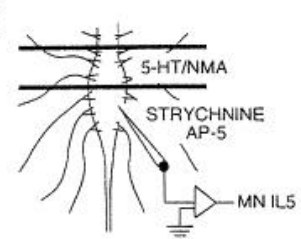

$\mathrm{A}_{2}$

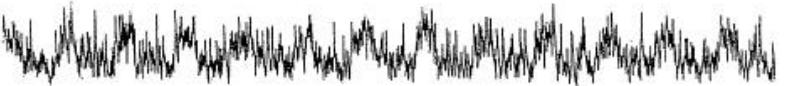

B2

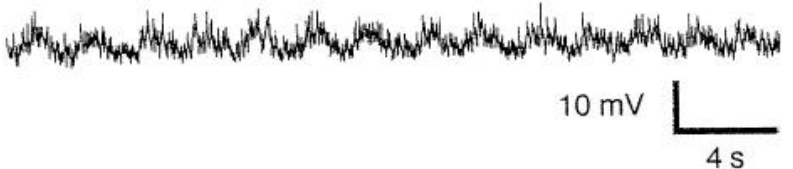

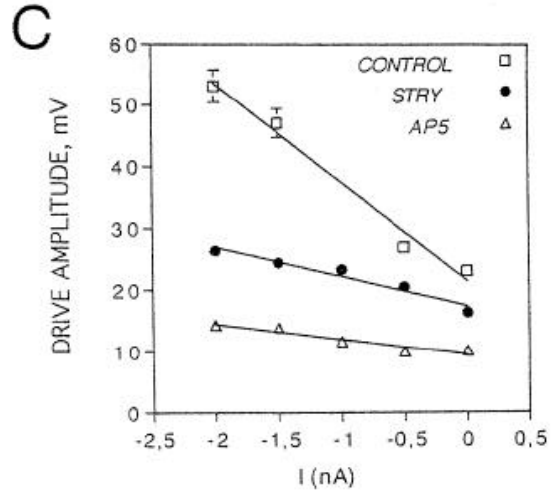

Figure 6. The excitatory synaptic drive onto motoneurons is partly blocked by AP-5. A, During bath application of 5-HT/NMDA to segments L1/L2, a motoneuron recorded in $\mathrm{L} 5$ (with a $\mathrm{KCl}$ electrode) in the presence of strychnine $\left(3 \times 10^{-6} \mathrm{M}\right)$ was rhythmically depolarized and spontaneously fired action potentials (membrane potential at bottom, $-68 \mathrm{mV})$. B, Adding AP-5 $\left(5 \times 10^{-5} \mathrm{M}\right)$ to the strychnine saline partially blocked the excitatory drive (membrane potential, $-70 \mathrm{mV}$ ). $C$, Changes in the drive amplitude. Under control conditions $(\square)$, the drive amplitude varied considerably $($ slope $=-16$ ) depending on the current injected to the motoneuron, whereas it was less sensitive to current (slope $=-5$ ) in the presence of strychnine $(\bullet)$. In the presence of AP-5 and strychnine $(\triangle)$, the drive was still less sensitive (slope $=-2$ ) to changes in the injected current. Error bar: SD, $n \geq 12$ at each point.

(bicuculline or pfaclofen) did not modify the phase relationships of the motor pattern (Cazalets et al., 1994). In the isolated spinal cord of newborn rat, Kudo et al. (1991) observed that strychnine triggered in-phase right and left activities. We have never been able to observe consistently any such in-phase pattern, however, because we always obtained a high-frequency rhythm (as in Fig.

\section{A1 CONTROL}
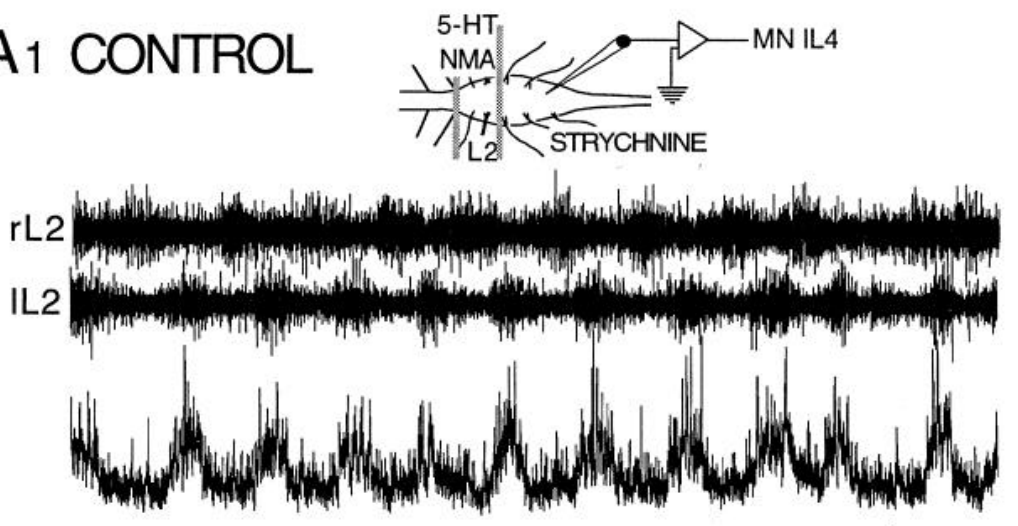

$2 \mathrm{mV}$

\section{A2 CNQX}
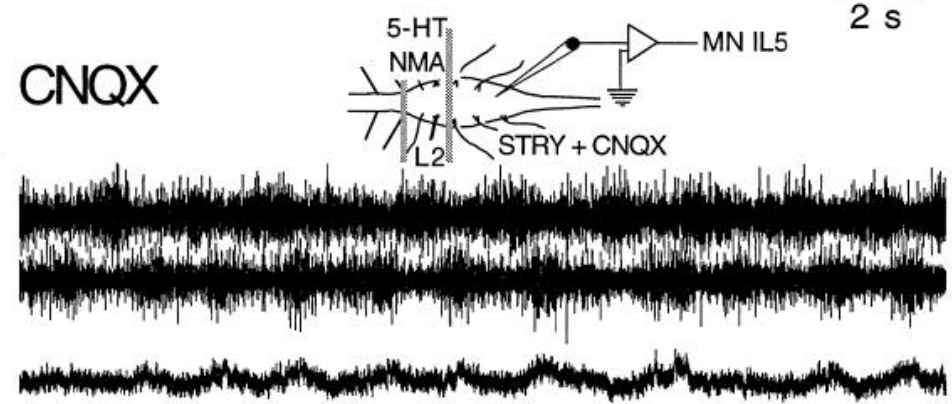

B1

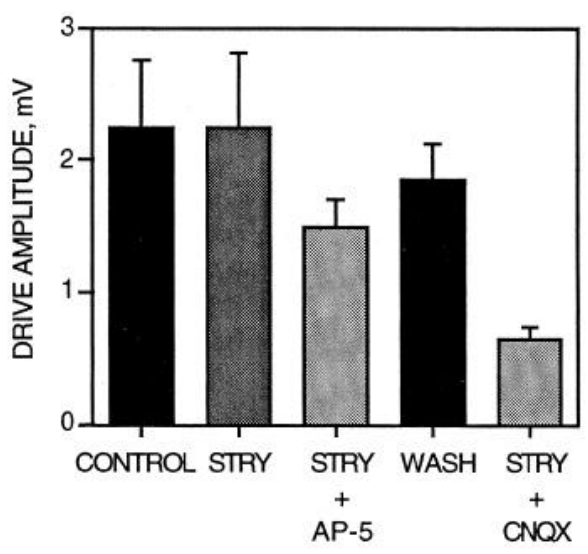

B2

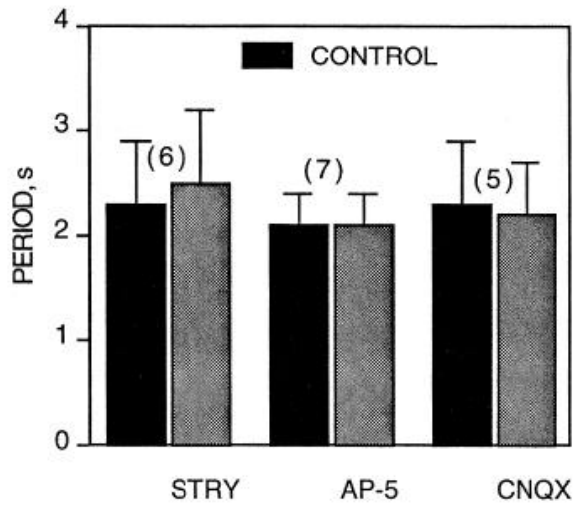

Figure 7. The excitatory synaptic drive onto motoneurons is partly blocked by CNQX. A, During bath application of 5-HT/NMDA to segments L1/L2, a motoneuron recorded in L4 (with a potassium acetate electrode) was rhythmically depolarized in the presence of strychnine $\left(3 \times 10^{-6} \mathrm{M} ; A 1\right.$, membrane potential at bottom, $-68 \mathrm{mV})$. The subsequent addition of CNQX $\left(5 \times 10^{-6} \mathrm{M} ; A 2\right.$, membrane potential at bottom, $\left.-69 \mathrm{mV}\right)$ to the strychnine saline considerably decreased the size of the EPSPs. B1, Plot of the changes elicited in the drive amplitude by successive bath application of strychnine, strychnine + AP-5, and strychnine + CNQX. $A$ and $B$ are from two different neurons. Measurements were performed during at least 10 successive cycles in each case. B2, Plot of the period value when strychnine, AP-5, or CNQX were bath-applied to the lower lumbar segments (L3-L6) during sequences of locomotor-like activity elicited by bath application of 5-HT/NMDA to segments L1/L2. The mean period was measured under control conditions immediately before or after the drug application. $n$, number of experiments. 
$4 C)$ that was not correlated with locomotor-like activity. Only in a few cases were some brief, erratic episodes of in-phase bursts of activity observed. The disruption of the motor activity observed in response to application of strychnine may have resulted, therefore, from what might be called a "network effect." Second, glycine also acts at the output of the spinal locomotor network, mediating the chloride-sensitive inhibition that this network exerts on the motoneurons (Fig. 5). This was confirmed indirectly by bicuculline's lack of effect on motoneuronal inhibition, which in our study was monitored directly by performing intracellular recordings (data not shown). A similar chloridc-sensitive rhythmic inhibition of the motoneurons has been observed in the lamprey (Russell and Wallén, 1983), the tadpole (Roberts et al., 1983, 1986; Soffe, 1987, 1989), and the cat (Orsal et al., 1986). In the adult cat, Pratt and Jordan (1987) have noted that intravenous injection of strychnine abolishes the inhibition exerted on motoneurons without modifying the locomotor rhythmicity.

\section{Involvement of excitatory arnino acids in the excitatory drive}

The role of excitatory amino acids in the initiation of locomotion has been investigated previously in various vertebrate species such as the lamprey (Cohen and Wallén, 1980; Grillner et al., 1981), the tadpole (Dale and Roberts, 1984), and the mudpuppy (Wheatley et al., 1992, 1994). In mammals, it was only quite recently that these substances were found to play a crucial role. The NMDA receptors were described first as activating the locomotor network in the newborn rat (Smith et al., 1988; Kudo et al., 1991; Cazalets et al., 1992), the cat (Douglas et al., 1993), and the rabbit (Fenaux et al., 1991). We further demonstrated that excitatory amino acids also act on the CPG via non-NMDA receptors (Cazalets et al., 1992; Sqalli-Houssaini et al., 1993b). The present study gives direct evidence that both types of receptors are also involved in the excitatory drive from the CPG to the motoneurons. The fact that AP-5 and CNQX reduced the synaptic drive demonstrates that the release of an endogenous excitatory amino acid activates the two types of receptors during locomotor-like activity. This contrasts with what occurs in the respiratory system, where direct information has also been obtained about the nature of the drive to the motoneurons. In this case, the respiratory drive to phrenic motoneurons is mediated only by non-NMDA receptors (Liu et al., 1990).

The respective contributions of the components to the drive, i.e., $-40 \%$ in the case of the NMDA and $60 \%$ in that of the non-NMDA components (see Results), are only approximations. The contribution of the NMDA receptors under our experimental conditions, however, is possibly underestimated because of the $\mathrm{Mg}^{2+}$ block. The possibility can be ruled out, however, that a cross-effect between the two antagonists occurred. Although CNQX may act on NMDA receptors via the glycine site, this requires much higher concentrations $(3 \times$ $10^{-5} \mathrm{M}$; Birch et al., 1988) than what we used to block the synaptic drive. With the antagonist concentrations used here, however, it can be assumed on the basis of two arguments that an almost complete blockade of the receptors was obtained: (1) the complete disappearance of the EPSPs elicited by the sensory afferent stimulation in the presence of AP-5 and CNQX (see Material and Methods, data not shown); and (2) the effect of AP-5 and CNQX on the locomotor-like rhythm (Cazalets et al., 1992; Sqalli-Houssaini et al., 1993b), which was abolished completely at these concentrations.

Figure 8 summarizes our conclusions about the organization of the spinal interactions that emerges from our results. The spinal

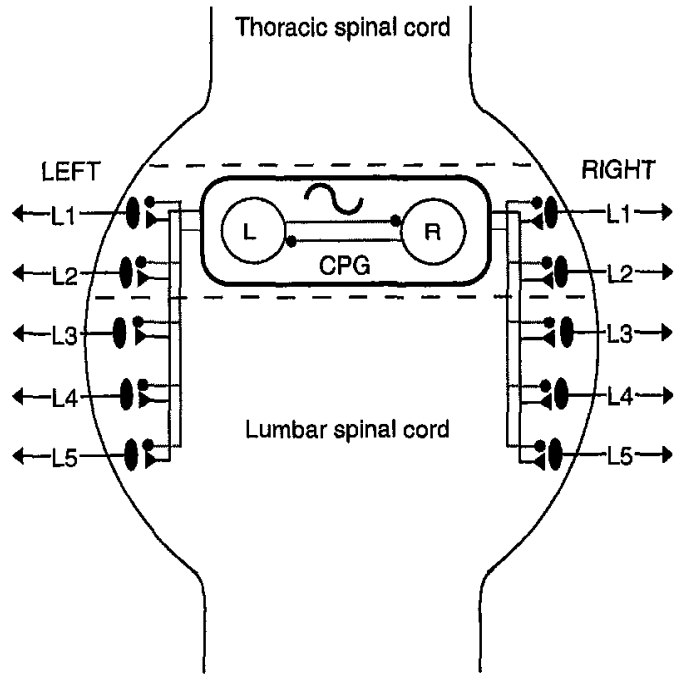

Figure 8. Diagram summarizing the connections between the spinal locomotor network located in L1/L2 and the motoneurons. (A) Excitalory amino acidergic connections; $(\bullet)$ glycinergic inhibitory connections.

locomotor network lies in the rostral part of the lumbar cord. Within this network, glycinergic inhibitions play a crucial role in the generation of a right and left alternating pattern. The locomotor network generates a biphasic output that is conveyed to the motoneurons, thus exerting both an inhibitory and an excitatory action on them. Although this model is rather different from those proposed, for example, in the case of the cat (Miller and Scott, 1977; see review by Jordan, 1983), there exist similarities between the synaptic inputs to the motoneurons, such as those involved in the mixed synaptic drive (IPSPs and EPSPs).

\section{REFERENCES}

Birch PJ, Grossman CJ, Hayes AG (1988) 6,7-Dinitro-quinoxaline-2,3dione and 6-nitro,7-cyano-quinoxaline-2,3-dion antagonise responses to NMDA in the rat spinal cord via an action at the strychnine-insensitive glycine receptor. Eur J Pharmacol 156:177-180.

Cazalets JR, Borde M, Clarac F (1995) Localization and organization of the central pattern generator for hindlimb locomotion in newborn rat. J Neurosci 15:4943-4951.

Cazalets JR, Sqalli-Houssaini Y, Clarac F (1992) Activation of the central pattern generators for locomotion by serotonin and excitatory amino acids in neonatal rat. J Physiol (Lond) 474:173-181.

Cazalets JR, Sqalli-Houssaini Y, Clarac F (1994) GABAergic inactivation of the central pattern generators for locomotion in isolated neonatal rat spinal cord. J Physiol (Lond) 455:187-204.

Cohen AH, Wallén P (1980) The neuronal correlate of locomotion in fish. "Fictive swimming" induced in an in vitro preparation of the lamprey spinal cord. Exp Brain Res 41:11-18.

Dale N, Roberts A (1984) Excitatory amino acid receptors in Xenopus embryo spinal cord and their role in the activation of swimming. $J$ Physiol (Lond) 348:527-543.

Douglas JR, Noga BR, Dai X, Jordan LM (1993) The effects of intrathecal administration of excitatory amino acid agonists and antagonists on the initiation of locomotion in the adult cal. J Neurusci 13:990-1000.

Fenaux F, Corio M, Palisses R, Viala D (1991) Effects of an NMDAreceptor antagonist, $\mathrm{MK}-801$, on central locomotor programming in the rabbit. Exp Brain Res 86:393-401.

Grillner S (1981) Control of locomotion in bipeds, tetrapods and fish. In: Handbook of physiology. The nervous system II (Brookhart JM, Mountcastle VB, eds), pp 1179-1236. Bethesda, MD: American Physiological Society.

Grillner S, Matsushima T (1991) The neural network underlying locomotion in lamprey, synaptic and cellular mechanisms. Neuron 7:1-15.

Grillner S, McClellan A, Sigvardt K, Wallén P, Wilén M (1981) Activation of NMDA-receptors elicits "fictive locomotion" in lamprey spinal cord in vitro. Acta Physiol Scand 113:549-551. 
Jahr CE, Yoshioka K (1986) Ia afferent excitation of motoneurones in the in vitro new-born rat spinal cord is selectively antagonized by kynurenate. J Physiol (Lond) 370:515-530.

Jordan LM (1983) Factors determining motoneuron rhythmicity during fictive locomotion. Symp Soc Exp Biol 37:423-444.

Jordan LM (1991) Brainstem and spinal cord mechanisms for the initiation of locomotion. In: Neurobiology basis of human locomotion (Shimamura M, Grillner S, Edgerton VR, eds), pp 3-20. Tokyo: Japanese Science Society.

Kiehn O, Kjaerulff O (1995) Spatiotemporal characteristics of 5-HT and dopamine-induced rhythmic hindlimb activity in the in vitro neonatal rat. $\mathbf{J}$ Neurophysiol, in press.

Kudo N, Ozaki S, Yamada T (1991) Ontogeny of rhythmic activity in the spinal cord of the rat. In: Neurobiological basis of human locomotion (Shimamura M, Grillner S, Edgerton VR, eds), pp 126-137. Tokyo: Japanese Science Society.

Liu G, Feldman JL, Smith JC (1990) Excitatory amino acid-mediated transmission of inspiratory drive to phrenic motoneurons. J Neurophysiol 64:423-436.

Miller S, Scott PD (1977) The spinal locomotor generator. Exp Brain Res 30:387-403.

Orsal D, Perret C, Cabelguen JM (1986) Evidence of rhythmic inhibitory synaptic influences in hindlimb motoneurons during fictive locomotion in the thalamic cat. Exp Brain Res 64:217-224.

Perret C (196) Synaptic influences contributing to the pattern of limb motoneuron activity during fictive locomotion in the cat. In: Neurobiology of vertebrate locomotion (Grillner S, Stein PSG, Stuart DG, Forssberg H, Herman RM, eds), pp 173-185. New York: MacMillan.

Pinco M, Lev-Tov A (1993) Synaptic excitation of $\alpha$-motoneurons by dorsal root afferents in the neonatal rat spinal cord. J Neurophysiol 70:406-417.

Pratt CA, Jordan LM (1987) Ia inhibitory interneurons and Renshaw cells as contributors to the spinal mechanisms of ficlive locomotion. J Neurophysiol 57:56-71.

Roberts A, Soffe SR, Clarke JDW, Dale N (1983) Initiation and control of swimming in amphibian embryos. Symp Soc Exp Biol 37:261-284.
Roberts A, Soffe SR, Dale N (1986) Spinal interneurones and swimming in frog embryos. In: Neurobiology of vertebrate locomotion (Grillner $S$ Stein PSG, Stuart DG, Forssberg H, Herman RM, eds), pp 279-306. New York: MacMillan.

Russell DF, Wallén P (1983) On the control of myotomal motoneurons during "fictive swimming" in the lamprey spinal cord in vitro. Acta Physiol Scand 117:161-170.

Shefchyk SJ, Jordan LM (1985) Excitatory and inhibitory postsynaptic potentials in $\alpha$-motoneurons produced during fictive locomotion by stimulation of the mesencephalic locomotor region. J Neurophysiol 53:1345-1355.

Soffe SR (1987) Ionic and pharmacological properties of reciprocal inhibition in Xenopus embryo motoneurons. J Physiol (Lond) 382:463-473.

Soffe SR (1989) Role of glycinergic inhibition and $N$-methyl-D-aspartate receptor mediated excitation in the locomotor rhythmicity of one half of the Xenopus embryo central nervous system. Eur $\mathbf{J}$ Neurosci 1:561-571.

Smith JC, Feldman JL Schmidt BJ (1988) Neural mechanisms generating locomotion studied in mammalian brain stem-spinal cord in vitro. FASEB J 2:2283-2288.

Sqalli-Houssaini Y, Cazalets JR, Clarac F (1991) A cooling/heating system for use with in vitro preparations: study of temperature effects on newborn rat rhythmic activities. J Neurosci Methods 39:131-139.

Sqalli-Houssaini Y, Cazalets JR, Clarac F (1993a) Oscillatory properties of the central pattern generator for locomotion in neonatal rats. J Neurophysiol 70:803-813.

Sqalli-Houssaini Y, Cazalets JR, Martini F, Clarac F (1993b) Induction of fictive locomotion by sulphur-containing amino acids in an in vitro newborn rat preparation. Eur J Neurosci 5:1226-1232.

Wheatley M, Edamura M, Stein RB (1992) A comparison of intact and in vitro locomotion in an adult amphibian. Exp Brain Res 88:609-614.

Wheatley M, Jovanovic K, Stein RB, Lawson V (1994) The activity of interneurons during locomotion in the in vitro necturus spinal cord. J Neurophysiol 71:2025-2032. 